\title{
Blocking AMPA receptor signalling by caroverine infusion does not affect counter-regulation of hypoglycaemia in healthy men
}

\author{
J. Klement • I. Pais • M. Hallschmid • C. Hubold • \\ A. Knispel • K. M. Oltmanns • B. Schultes • J. Born • \\ A. Peters
}

Received: 9 December 2008 / Accepted: 3 March 2009/Published online: 3 April 2009

(C) Springer-Verlag 2009

\begin{abstract}
Aims/hypothesis Glutamatergic pathways are assumed to play a critical role in the hormonal stress response to hypoglycaemia. In rats, glutamate signalling at the amino3-hydroxy-5-methyl-4-isoxazol propionate (AMPA) receptor contributes to hormone release induced by behavioural stressors. We hypothesised that blocking the AMPA receptor by caroverine in healthy men would impair their perception of neuroglycopenia and thereby diminish hormonal counter-regulation as well as symptoms of hypoglycaemia, as a model of stress.

Methods In a balanced double-blind study, two hypoglycaemic clamp sessions (mean blood glucose $2.4 \mathrm{mmol} / \mathrm{l}$ for $50 \mathrm{~min}$ ) were performed in ten healthy men during intravenous administration of $80 \mathrm{mg}$ caroverine or placebo. We assessed concentrations of counter-regulatory hormones as well as subjective symptoms related to hypoglycaemia.
\end{abstract}

J. Klement and I. Pais contributed equally to this study.

J. Klement $(\varangle) \cdot$ I. Pais $\cdot$ C. Hubold $\cdot$ A. Knispel $\cdot$ A. Peters Department of Internal Medicine I, University of Luebeck, Ratzeburger Allee 160,

23538 Luebeck, Germany

e-mail: Johanna.Klement@uk-sh.de

M. Hallschmid $\cdot$ K. M. Oltmanns $\cdot$ J. Born

Department of Neuroendocrinology, University of Luebeck,

Luebeck, Germany

K. M. Oltmanns

Department of Psychiatry and Psychotherapy,

University of Luebeck,

Luebeck, Germany

B. Schultes

Obesity Center, Kantonsspital St Gallen,

St Gallen, Switzerland
Results AMPA receptor antagonisation by caroverine did not influence the perception of neuroglycopenic and autonomic hypoglycaemia-associated symptoms $(p>0.39$ for all). Notwithstanding, caroverine did increase basal and counter-regulatory glucagon secretion $(p<0.002)$ and slightly enhanced counter-regulatory growth hormone concentrations $(p=0.07)$. Counter-regulatory release of ACTH, cortisol, adrenaline (epinephrine) and noradrenaline (norepinephrine) did not differ between conditions $(p>0.11$ for all).

Conclusions/interpretation Antagonising AMPA receptor signalling by caroverine infusion failed to diminish and even slightly amplified counter-regulatory hormone release during hypoglycaemia in healthy men. The discrepancy with previous findings in rats may be due to different dosages or administration routes and calls for further investigations on the role of AMPA receptor signalling in hypoglycaemia counter-regulation in humans.

Keywords AMPA receptor - Caroverine - Glutamate receptor · Hormonal counter-regulation · Hypoglycaemia . Neuroglycopenic symptoms

\author{
Abbreviations \\ AMPA Amino-3-hydroxy-5-methyl-4-isoxazol \\ propionate \\ HPA Hypothalamic-pituitary-adrenal \\ NMDA $N$-methyl-D-aspartate
}

\section{Introduction}

The physiological mechanisms underlying the counterregulation of hypoglycaemia are of great clinical impor- 
tance because counter-regulatory failure is a severe side effect in the insulin treatment of patients with type 1 and type 2 diabetes mellitus. Neuronal pathways using the amino acid transmitter glutamate have been shown to contribute to neuroendocrine counter-regulation following insulin-induced hypoglycaemia [1], supporting the assumption that in stressful conditions like hypoglycaemia, glutamate signalling is essential for activation of the hypothalamic-pituitaryadrenal (HPA) system, which allocates glucose to the brain [2]. Accordingly, blocking the glutamatergic $N$-methyl-Daspartate (NMDA) receptor by intracerebral administration of memantine has been found to decrease the counter-regulatory hormonal response to insulin-induced hypoglycaemia in dogs [3]. However, our own observations indicate that antagonising NMDA receptors by oral pre-treatment with memantine does not decrease the counter-regulatory response to hypoglycaemia in humans [4]. As the glutamate signal is conveyed by amino-3-hydroxy-5-methyl-4-isoxazol propionate (AMPA) receptor subtypes, whereas NMDA receptors are thought to play a role in inducing or modulating the plasticity of AMPA receptor-mediated transmission, the above finding raises the question as to what role AMPA receptors play in the acute neuroendocrine and neurocognitive response to hypoglycaemia in humans. In rats, pharmacological blockade of AMPA receptors diminished the neuroendocrine response to immobilisation stress [5-7]. Against this background, we assessed neuroglycopenic and autonomic symptoms, and neuroendocrine responses to insulin-induced hypoglycaemia in healthy men after pretreatment with the AMPA receptor antagonist caroverine.

\section{Methods}

Participants The participants in our experiments were ten healthy men with a mean $( \pm$ SEM) age of $24.4 \pm 0.95$ years and a mean $\left( \pm\right.$ SEM) BMI of $21.6 \pm 0.69 \mathrm{~kg} / \mathrm{m}^{2}$. Exclusion criteria were any physical or mental disease and abuse of nicotine, alcohol or drugs. The study was approved by the local ethics committee and all participants gave written informed consent.

Experimental design and hypoglycaemic clamp procedure Each participant underwent two hypoglycaemic clamps separated by an interval of at least 4 weeks. Participants received $80 \mathrm{mg}$ of the AMPA receptor antagonist caroverine (Phafag, Schaanwald, Liechtenstein) intravenously in one experimental condition and placebo $(0.9 \%$ [wt $/ \mathrm{vol}$.] saline solution) in the other. Data on the placebo condition are derived from a parallel study [4], where all details regarding the experimental set-up can be found. The study was handled in a double-blind fashion with the order of conditions balanced across participants.
On days when experiments were performed, participants reported to the medical research unit at 06:45 hours after an overnight fast of at least $10 \mathrm{~h}$. At 07:00 hours, participants received a bolus of $16 \mathrm{mg}$ caroverine, followed by constant infusion at a rate of $25.6 \mathrm{mg} / \mathrm{h}$ for the next $150 \mathrm{~min}$, resulting in a total amount of $80 \mathrm{mg}$ caroverine. This dose rate exerts significant effects on human brain function as previously indicated by EEG recordings and cognitive tests [8]. In the placebo condition, saline solution was administered. The initial $30 \mathrm{~min}$ of caroverine and placebo infusion before initiation of the hypoglycaemic clamp procedure are referred to as the euglycaemic period $(t=-30$ to $t=0)$. At 07:30 hours $(t=0)$, i.e. after the 30 -min euglycaemic period, participants received a bolus injection of $10 \mathrm{mU} / \mathrm{kg}$ body weight of human insulin (Aventis, Bad Soden, Germany), followed by constant-rate infusion of $1.5 \mathrm{mU} \mathrm{kg} \mathrm{min}^{-1}$ insulin for the next $120 \mathrm{~min}$. Blood glucose levels were measured every 5 min (HemoCue, Ängelholm, Sweden) and a solution of $20 \%$ glucose was infused simultaneously at variable rates to maintain the target blood glucose level of $2.4 \mathrm{mmol} / \mathrm{l}$ during the hypoglycaemic plateau. This plateau was reached after $70 \mathrm{~min}$ of insulin infusion (at 08:40 hours) and maintained for $50 \mathrm{~min}$ (until 09:30 hours). Thereafter, infusion of insulin and caroverine/placebo was stopped and blood glucose levels returned to euglycaemic concentrations. Blood samples for determination of insulin,

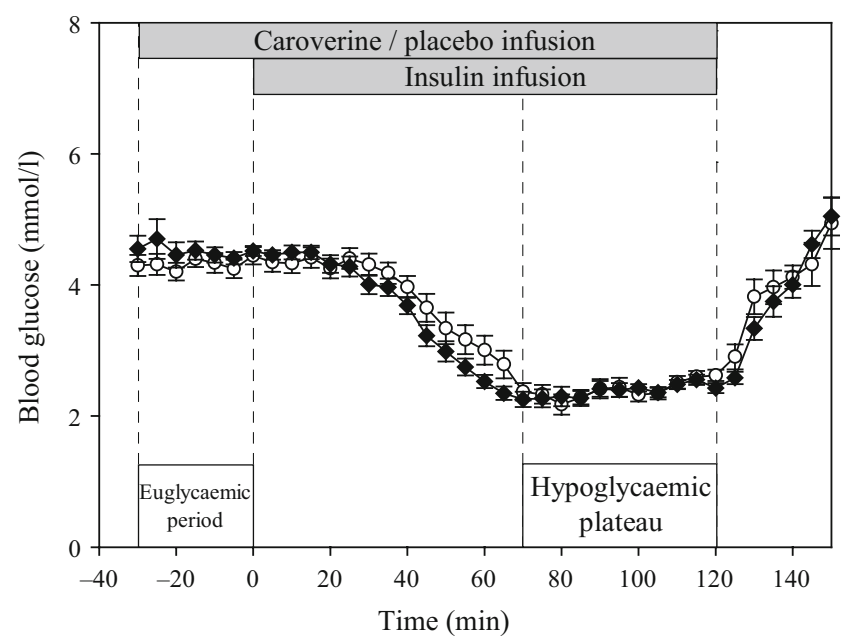

Fig. 1 Blood glucose concentrations (mean \pm SEM) during caroverine and placebo infusion. Caroverine $(16 \mathrm{mg}$ bolus followed by constant infusion at $25.6 \mathrm{mg} / \mathrm{h}$ for $150 \mathrm{~min}$; black diamonds) and placebo infusion (white circles) started at the beginning of a $30 \mathrm{~min}$ euglycaemic period ( -30 to $0 \mathrm{~min}$ ) followed by a bolus insulin injection of $10 \mathrm{mU} / \mathrm{kg}$ body weight and a subsequent constant-rate infusion of $1.5 \mathrm{mU} \mathrm{kg}^{-1} \mathrm{~min}^{-1}$ for the next $120 \mathrm{~min}$. The target glucose level was reached $70 \mathrm{~min}$ after onset of the insulin infusion and maintained for another $50 \mathrm{~min}$. After $120 \mathrm{~min}$, insulin and caroverine infusions were stopped and euglycaemic blood glucose concentrations were restored. Key time-points are indicated by dotted vertical lines 
Fig. 2 Plasma or serum concentrations of (a) ACTH, (b) cortisol, (c) adrenaline, (d) noradrenaline, (e) growth hormone and (f) glucagon (mean \pm SEM) during caroverine and placebo infusion. Caroverine (black circles) and placebo (white circles) infusion was as shown in Fig. 1. The target glucose level was reached 70 min after onset of the insulin infusion and maintained for another $50 \mathrm{~min}$. The euglycaemic period and hypoglycaemic plateau are indicated by dotted vertical lines. ${ }^{*} p<0.05$ for comparison between conditions
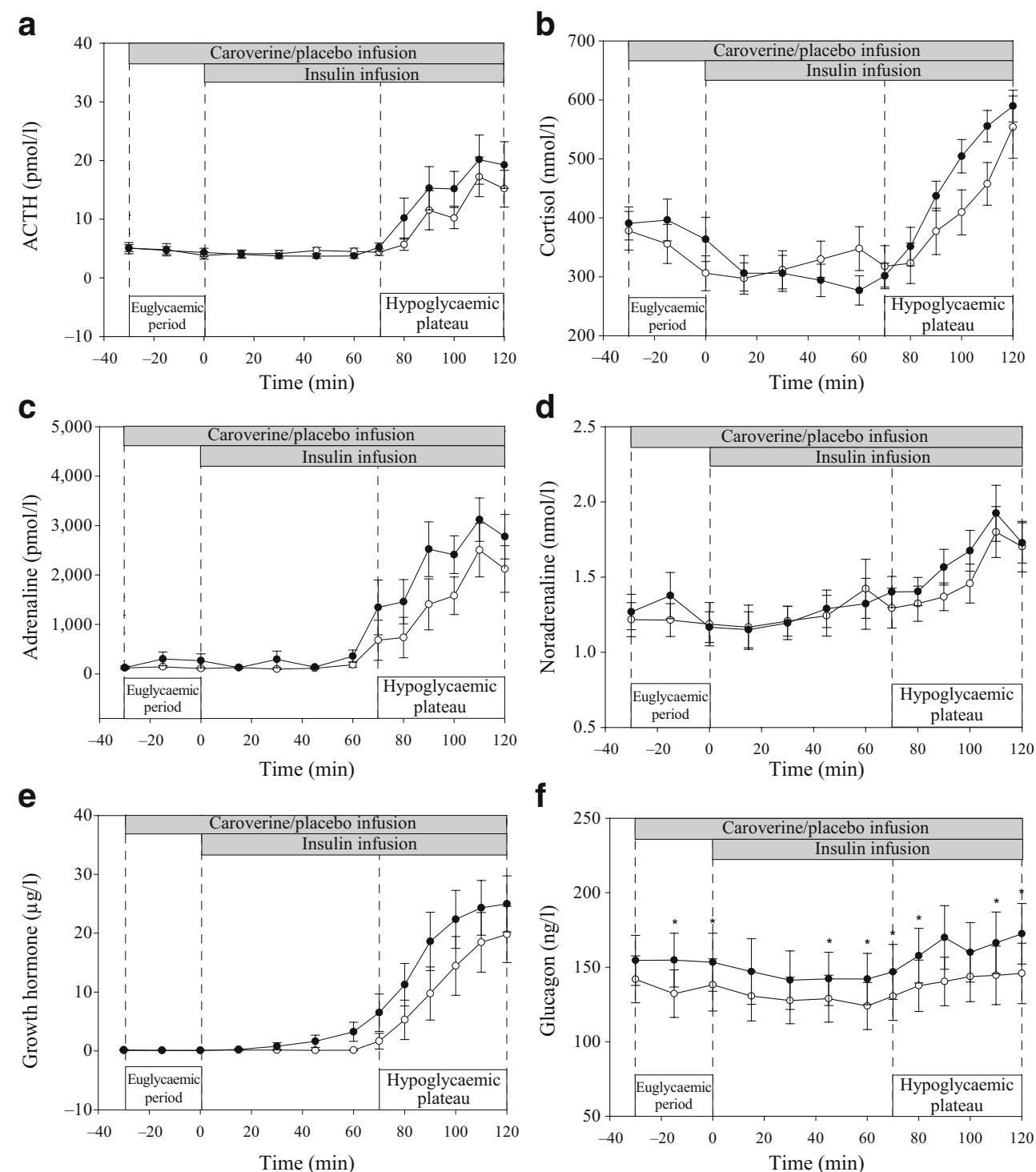

C-peptide, ACTH, cortisol, catecholamine, growth hormone and glucagon levels were drawn at 15 min intervals during the euglycaemic period and before the hypoglycaemic plateau, and every $10 \mathrm{~min}$ during that plateau. Participants rated autonomic and neuroglycopenic symptoms on a questionnaire presented $30 \mathrm{~min}$ before the insulin infusion $(t=-30)$ and at 10,30, 80, 100, 120 and $160 \mathrm{~min}$ after initiation of insulin infusion, as previously described in detail [4].

Statistical analysis Data are presented as means \pm SEM. Analysis is based on ANOVA for repeated measures, including the factors 'treatment' (caroverine vs placebo) and 'time' (time-points of data collection). Where necessary, degrees of freedom were corrected using the Greenhouse-Geisser procedure. For pair-wise comparisons, Student's paired-samples $t$ test was applied. A $p$ value of $<0.05$ was considered significant.

\section{Results}

Mean blood glucose levels were similar in both conditions, declining from the euglycaemic period (placebo $4.30 \pm$ $0.13 \mathrm{mmol} / \mathrm{l}$, caroverine $4.52 \pm 0.16 \mathrm{mmol} / \mathrm{l}$ ) to the hypoglycaemic plateau reached $70 \mathrm{~min}$ after onset of insulin infusion (placebo $2.40 \pm 0.07 \mathrm{mmol} / \mathrm{l}$, caroverine $2.37 \pm 0.08 \mathrm{mmol} / \mathrm{l}$; $p<0.001$ for time, $p>0.18$ for all comparisons between conditions) (Fig. 1). Glucose infusion rates increased during hypoglycaemia but did not differ between conditions ( $p>$ $0.14)$. Neuroglycopenic as well as autonomic symptom scores increased during hypoglycaemia from $7.0 \pm 2.13$ vs $5.6 \pm 1.38$ (placebo vs caroverine, euglycaemic period) and $8.8 \pm 2.07$ vs $5.5 \pm 1.15$ respectively to $11.30 \pm 2.17$ vs $11.87 \pm$ 2.09 and $14.83 \pm 2.77$ vs $14.76 \pm 2.86$ respectively, but these increases were not affected by treatment $(p>0.59)$.

In response to hypoglycaemia, circulating levels of $\mathrm{ACTH}$, cortisol, adrenaline (epinephrine) and noradrenaline 
(norepinephrine) increased ( $p<0.001$ for time effect for all) (Fig. 2a-d) without any differences between conditions (all $p>0.11$ ). In contrast, the growth hormone response to hypoglycaemia tended to display higher levels in the caroverine than in the placebo condition $(p=0.07$ for treatment, $p=0.24$ for treatment $\times$ time) (Fig. 2e). Moreover, glucagon levels during the euglycaemic period as well as during hypoglycaemia were overall distinctly higher in the caroverine than in the placebo condition $(p<0.002$ for treatment; $p>0.65$ for treatment $\times$ time) (Fig. 2 f).

\section{Discussion}

Blocking AMPA receptor signalling by caroverine does not essentially alter the release of counter-regulatory hormones and the perception of neuroglycopenic symptoms during $50 \mathrm{~min}$ of pronounced hypoglycaemia in healthy men. Contrary to our hypothesis, there was even a weak indication of increased growth hormone secretion during the hypoglycaemic plateau. In rats, intracerebral stimulation of AMPA receptors induces elevations of plasma ACTH [6] and their antagonisation inhibits ACTH and prolactin secretion in response to immobilisation stress [7]. At first glance, these results conflict with our observations of unchanged HPA axis activity. However, different stress stimuli have been shown to provoke stressor-specific counter-regulatory responses that are possibly mediated via different central nervous pathways. Whereas the blockade of AMPA and NMDA receptors suppresses the ACTH response to immobilisation stress in rats, the neuroendocrine response to foot shock or ether stress is not affected by blockade of these receptors [5]. Our finding that blocking AMPA receptors by caroverine does not alter the neuroendocrine counter-regulatory response to hypoglycaemia suggests that in humans neuroendocrine responses to this specific metabolic stressor are mediated via AMPA-independent pathways. Also, species-dependent differences cannot be ruled out and must be taken into consideration.

Differences in the administration route between animal and human studies may also be crucial for the effects of glutamatergic interventions. In our experiments, caroverine was administered intravenously, whereas most animal studies used the intracerebroventricular or intracerebral route $[6,7]$. It is conceivable that peripherally administered caroverine does not reach the hypothalamus in sufficient quantities or, additionally, that AMPA receptor antagonism in other brain areas counteracts the hypothalamic effects of caroverine on neuroendocrine systems. The high relevance of the administration mode is supported by findings in mice, where intraperitoneal administration of the AMPA receptor antagonist cyano-nitro-quinoxaline-dione in- creased corticosterone levels, while intracerebroventricular application had reverse effects [9]. Also, different dose regimens of caroverine administration may yield diverging results. However, the intravenous administration of $80 \mathrm{mg}$ caroverine as used in our study has previously been shown to induce significant central nervous effects [8], while higher doses are associated with the risk of biasing side effects, especially sedation.

Unexpectedly, caroverine administration increased glucagon concentrations during the euglycaemic period as well as during hypoglycaemia. In contrast, in vivo blockade of AMPA receptors on pancreatic alpha cells induces negative autocrine feedback on glucagon secretion in mice [10]. One possible mechanism behind the surprising increase in glucagon secretion in our study might be that central nervous AMPA blockade by caroverine stimulates autonomic pathways that are known to trigger glucagon release during hypoglycaemia and that such an effect overrode the presumably suppressive effect of systemic AMPA receptor blockade on glucagon secretion.

In sum, our results in healthy men show that peripheral administration of an AMPA receptor blocker does not modify the neuroendocrine response to hypoglycaemia. Future in vivo studies focusing on different dose regimens and possibly also administration routes are needed to provide further insight into the role of glutamatergic AMPA receptor signalling in hypoglycaemia counter-regulation.

Acknowledgements We thank K.-U. Duysen, K. Kurwahn, I. v. Lützau and C. Otten for expert technical assistance. This work was supported by the German Research Foundation (DFG; KFO-126).

Duality of interest The authors declare that there is no duality of interest associated with this manuscript.

\section{References}

1. Tong Q, Ye C, McCrimmon RJ et al (2007) Synaptic glutamate release by ventromedial hypothalamic neurons is part of the neurocircuitry that prevents hypoglycemia. Cell Metab 5:383-393

2. Peters A, Pellerin L, Dallman MF et al (2007) Causes of obesity: looking beyond the hypothalamus. Prog Neurobiol 81:61-88

3. Molina PE, Abumrad NN (2001) Contribution of excitatory amino acids to hypoglycemic counter-regulation. Brain Res 899:201-208

4. Pais I, Hubold C, Hallschmid M et al (2008) Blocking NMDA receptor signaling does not decrease hormonal counterregulation to hypoglycemia in humans. Psychoneuroendocrinology 33:10691076

5. Zelena D, Makara GB, Jezova D (1999) Simultaneous blockade of two glutamate receptor subtypes (NMDA and AMPA) results in stressor-specific inhibition of prolactin and corticotropin release. Neuroendocrinology 69:316-323

6. Umegaki H, Yamamoto A, Suzuki Y, Iguchi A (2006) Stimulation of the hippocampal glutamate receptor systems induces stress-like responses. Neuro Endocrinol Lett 27:339-343

7. Kusakawa S, Tohei A, Jaroenporn S, Watanabe G, Taya K (2007) Inhibition of stress-induced adrenocorticotropin and prolactin 
secretion mediating hypophysiotropic factors by antagonist of AMPA type glutamate receptor. J Reprod Dev 53:545-554

8. Saletu B, Grunberger J, Anderer P, Linzmayer L, Konig P (1995) Acute central effects of the calcium channel blocker and antiglutamatergic drug caroverine. Double-blind, placebocontrolled, EEG mapping and psychometric studies after intravenous and oral administration. Arzneimittelforschung 45:217-229
9. Kim DH, Moon YS, Jung JS, Suh HW, Song DK (2003) Routedependent effects of the non-NMDA receptor antagonist CNQX on plasma corticosterone levels in mice. Prog Neuropsychopharmacol Biol Psychiatry 27:1055-1058

10. Cabrera O, Jacques-Silva MC, Speier S et al (2008) Glutamate is a positive autocrine signal for glucagon release. Cell Metab 7:545554 